\title{
Dark Energy: Nature and Robustness
}

\author{
A. Blanchard ${ }^{1}$ and Y. Zolnierowski ${ }^{1,2}$ \\ 1 LAOMP, 14, Av. E.Belin, 31400 Toulouse, France \\ alain.blanchard@ast. obs-mip.fr \\ 2 LAPP, IN2P3-C.N.R.S., B.P. 110, 74941 Annecy-le-Vieux cédex, France
}

Summary. Supernovae have provided the evidence for the existence of a dominant dark energy component of the Universe. The commonly accepted form of such a component is the quintessence. Here, we show that the possible nature of this component is rather well constrained by combining the various existing observational cosmological data. However, relaxing some of the various hypothesis can lead to somewhat different results.

\section{Introduction}

In a couple of years the so-called concordance model, i.e. $\Omega_{M} \sim 0.3 \Omega_{\Lambda}=$ 1. $-\Omega_{M} \sim 0.7$ and $H_{0} \sim 72 \mathrm{~km} / \mathrm{s} / \mathrm{Mpc}$ has become almost the standard model of modern cosmology. Its ability to reproduce several major observations of cosmological relevance is actually remarkable. In addition the WMAP signal is fully consistent with such a model which has therefore gained in strength. However, it requires the introduction of a non-zero cosmological constant which represents the actual dominant contribution to the density of the Universe. Although such a possibility has been advocating by Einstein for cosmological purpose since 1917, cosmologists have been reluctant to take seriously into account such a term during the last eighty years! The question of whether there is an actual non-zero cosmological constant (or something similar), dominating the density of the Universe and producing its acceleration is certainly one of the most important and surprising question of modern physics. Here we would like to point out that direct evidence for a cosmological constant is very limited.

\section{The Cosmological Constant: a Strange Object}

The presence of a positive cosmological constant dominating the density of the Universe has rather dramatic consequences: the vacuum is actually the dominant component of the density of the Universe with a negative gravitational force inducing acceleration of the Universe. Indeed, within general relativity the source of gravity is: 


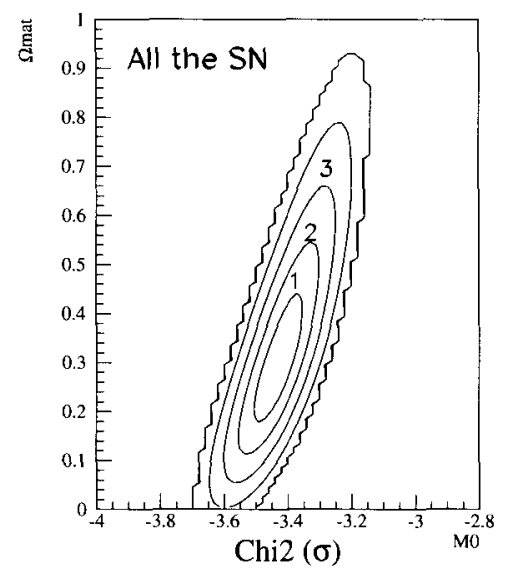

Fig. 1. Classical 1, 2, $3 \sigma$ contours in the plane $\Omega_{M}, \Omega_{\lambda}$ from the Hubble diagram of SNIa using the SCP supernovae set (Perlmutter et al., 1999).

$$
\rho+\frac{3 p}{c^{2}}
$$

$\rho$ being the density and $p$ the pressure. The vacuum has the following equation of state:

$$
p=-\rho c^{2}
$$

leading to a negative source of gravity. A first puzzling aspect of the concordance model is that we are at a very specific period of the Universe at which the vacuum starts to dominate the density of the Universe. For instance at the typical redshift of distant supernovae $(z \sim 0.5)$ the cosmological constant is not dominant any more over matter density. At redshift $z \sim 1$, the matter density parameter is already $\Omega_{m} \sim 0.75$. So that supernovae hunters $[7,8]$ were lucky enough that the acceleration was not so obvious that it will have been noticed from first cosmological observations, and that still the cosmological constant is not small enough to have escape observational detection! Whether this argument is a valid concern or not is certainly subject to discussion, but it should be remembered that this type of concern leads to one of the original argument in Guth's paper [4] to advocate inflation.

\section{Quintessence}

One of the physically motivated alternatives to the cosmological constant is known as quintessence. In this scenario it is assumed that a scalar field is actually dominating the density of the Universe [6, 12]. No better idea has been produced since, and the quintessence scenario is very popular, as 

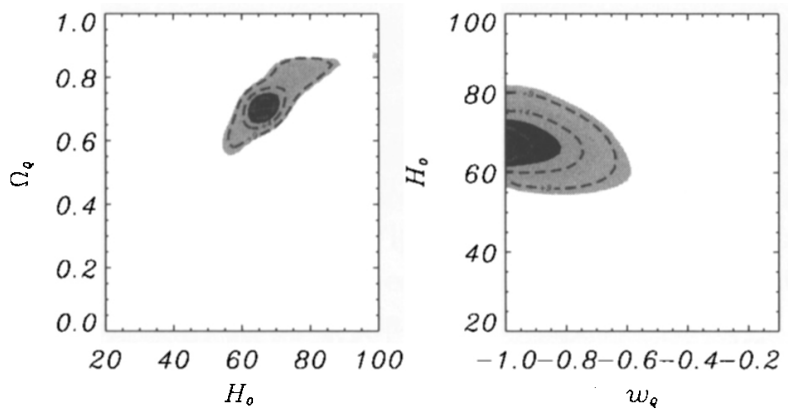

Fig. 2. $1,2,3 \sigma$ contours in the planes $H_{0}, \Omega_{M}$ and $w_{Q}, H_{0}$ from combining cosmological constraints including the Archeops CMB data [1].

being the only reasonable explanation of the acceleration of the Universe. The density of an homogenous scalar field is given by:

$$
\rho_{Q}=\frac{1}{2} \dot{\Phi}^{2}+V(\Phi)
$$

while the pressure is:

$$
p_{Q}=\frac{1}{2} \dot{\Phi}^{2}-V(\Phi)
$$

Such a field allows an arbitrary equation of state:

$$
p_{Q}=w(z) \rho_{Q}
$$

which is specified once the potential $V(\Phi)$ is given. Usually, $-1 \leq w$, although the region $w<-1$ is not forbidden. If $w<-1 / 3$ the expansion is accelerating. However, the various cosmological constraints (HST Hubble constant, Hubble diagram of distant supernovae, CMB, amplitude of matter fluctuations from clusters) allow to set severe constraints on this type of scenario, as shown on Fig. 2 [1]. The addition of the new WMAP data results in only a slight improvement [10]. This is an illustration of the fact that the standard scenario is very tightly constrained by existing data of cosmological relevance.

\section{Casting Doubts on the Concordance Model}

However, most of observational constraints used are subject to either some doubts from an observational point of view or to different interpretation when some simple hypotheses are relaxed. Moreover the concordance model conflicts with the observed abundance of high redshift clusters as analyzed in [2]. Probably the most important point is to remember that the CMB fluctuations, as observed by WMAP, do not require the introduction of a cosmological constant provided that the single power law assumption is relaxed. 

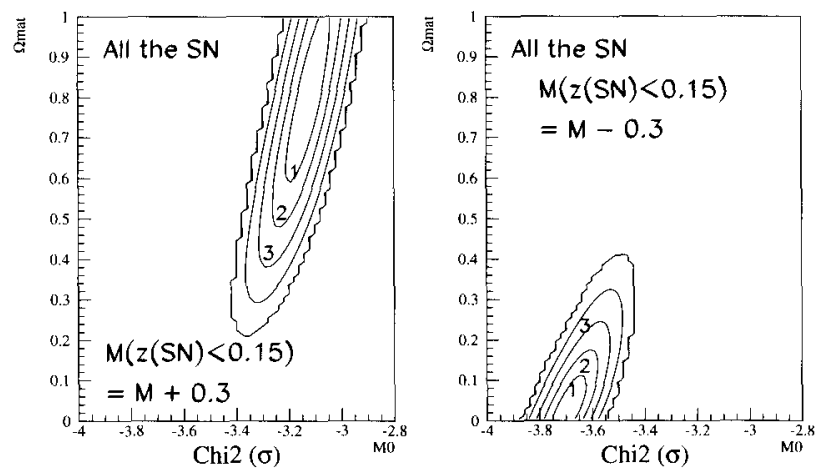

Fig. 3. Same contours than in figure 1, but after shifting the magnitude of distant $\mathrm{SNIa}(>0.15)$ by $\pm 0.3 \mathrm{mag}$.

Furthermore, the introduction of a additional small amount of dark matter, $\Omega_{D M} \sim 0.1$, which does not cluster on small scale, like massive neutrinos with $m_{\nu} \sim 1 \mathrm{eV}$ or quintessence with $w \sim 0$, allows to reproduce the matter amplitude $\left(\sigma_{8} \sim 0.55\right.$ for $\left.\Omega_{M}=1\right)$; such models then reproduce large scale structure data as well as a concordance model. One may consider that such a model is not as simple as the concordance, but there is some subjectivity in such an appreciation. One can alternatively consider that the introduction of a cosmological constant is extremely ugly.

\subsection{Concerns with the Interpretation of Supernovae Hubble Diagram}

It is important to remember that the only direct evidence for an accelerating Universe comes from the observational fact that distant supernovae appear fainter than those observed at low redshift. The amplitude of this dimming is not large: the signal is between 0.3 and 0.5 mag. Let us examine two possible concerns here: dust extinction [9] and selection biases. A first surprising result is that the High $\mathrm{Z}$ team found a couple of distant supernova with $B-V \sim-0.2$ the average being clearly bluer than their low redshift counterpart [5]. At first, this could be interpreted as an indication that distant are less dusty. As the color indices differ by something of the order of $0.1 \mathrm{mag}$, it would mean that distant SNIa are $\sim 0.3-0.5$ intrinsically brighter, pushing cosmological density parameter down to zero (see Fig. 2). However, the recent observation of [11] indicates no clear statistical difference in the average color of distant supernovae. Furthermore, local supernovae are never as blue as those present in the High $\mathrm{Z}$ team sample, even when their dust content is estimated to be zero. This therefore suggests a color evolution which is compensated by a larger dust extinction. So the apparent identical color hide a large dust extinction. This would therefore imply that intrinsic evolution 
is hiding the greater dust obscuration. This is possible without requiring an exact cancelation given the large dispersion of existing data on SNIa. In such a case distant SNIa might well be fainter because of a larger dust extinction and not because of cosmological dimming. Assuming that SNIa with $z>0.15$ are dimmed by $0.3 \mathrm{mag}$ by such a process essentially cancel the need for a non-zero cosmological constant (see Fig. 3). Finally let us turn to the possible selection biases. One is first concerned by the fact that distant supernovae might be affected by the standard Malmquist bias:

$$
\Delta m \approx 1.4 \sigma^{2}
$$

Estimation of the amplitude of this bias is generally performed on the basis of the intrinsic dispersion of the luminosity after correction of the correlation between light curve and intrinsic luminosity. Taking a more conservative point of view and using the dispersion of the population implies that the amplitude of the possible bias could be:

$$
\Delta m \approx 1.4(0.3-0.5)^{2} \approx 0.125-0.35
$$

meaning that selection bias could be a real concern. At first look it seems that this bias if affecting the distant supernovae, would imply that the average population is intrinsically fainter. However, the procedure to identify distant SNIa is such that the correlation between peak magnitude and magnitude detection is small, therefore distant supernovae are likely to be weakly affected by this bias while local supernovae are more probably affected [7]. Again this would mean that distant SNIa appear fainter just because of selection biases, not because of cosmological dimming.

\section{Conclusions}

As we have summarized, although the concordance model is certainly the simplest way to reproduce a large set of existing data of cosmological relevance, direct evidence for the existence of cosmological constant is only coming from Supernovae Hubble diagram of SNIa, with a signal which is of the order of the intrinsic dispersion of the population. This means that moderate biases or/and astrophysical effects may significantly alter the significance of this result.

\section{References}

1. A. Benoît et al. : Astron. Astrophys. 399, L25 (2003)

2. A. Blanchard, R. Sadat, J. Bartlett, M. Le Dour: Astron. Astrophys. 412, $35(2000)$ 
3. A. Blanchard, M. Douspis, M. Rowan-Robinson, S. Sarkar: Astron. Astrophys. 412, 35 (2003)

4. A. Guth: Phys. Rev. D 23, 347 (1981)

5. B. Leibundgut: Ann. Rev. Astron. Astrophys. 39, 67 (2001)

6. P.J.E. Peebles, B. Ratra: Astrophys. J. Lett. 325, L17 (1998)

7. S. Perlmutter et al. : Astrophys. J. 517, 565 (1999)

8. A.G. Riess et al. : Astron. J. 332, 252 (2002)

9. M. Rowan-Robinson: Mon. Not. R. Astron. Soc. 332, 252 (2002)

10. D.N. Spergel et al. : Astrophys. J. Suppl. 148, 175 (2003)

11. M. Sullivan et al. : Mon. Not. R. Astron. Soc. 340, 1057 (2003)

12. C. Wetterich: Nuc. Phys. B302, 668 (1988) 